Research Article

\title{
One-Step Biosynthesis of Gold Nanoparticles Using Natural Reductive Extracts
}

\author{
Ibraheem Jaleel Ibraheem ${ }^{1}$, Khalil Thabit Hassan ${ }^{2}$, Hameed Hussein Ali $^{1}$, Ahmed Salman Obaid ${ }^{2}$ \\ ${ }^{1}$ Department of Chemistry, College of Science, University Of Anbar, Ramadi 30001, Iraq. \\ ${ }^{2}$ Department of Physics, College of Science, University Of Anbar, Ramadi 30001, Iraq 0000-0002-2902-6998. \\ Corresponding authors. E-mail: sc.khalil_alftyan@uoanbar.edu.iq
}

Received: May 16, 2020; Accepted: Aug. 13, 2020; Published: Nov. 25, 2020

Citation: Ibraheem Jaleel Ibraheem, Khalil Thabit Hassan, Hameed Hussein Ali, and Ahmed Salman Obaid, One-Step Biosynthesis of Gold Nanoparticles Using Natural Reductive Extracts. Nano Biomed. Eng., 2020, 12(4): 33 I-337.

DOI: 10.5101/nbe.v12i4.p331-337.

\begin{abstract}
The green chemical synthesis of nanoparticles was extensively employed in the promotion of the fabrication of nanoparticles. In the present work, a single-step biosynthetic eco-friendly method was utilised to synthesise Au NPs using leave aqueous extracts of Piper nigrum (PN), Ziziphus spinachristi (ZSC), and Eucalyptus globulus (EUCGLO). The extract played the role of the reducing and capping agents where the volume ratios of aqueous extract $(100 \mathrm{ppm})$ to the aqueous solution of $\mathrm{HAuCl}_{4} \cdot 4 \mathrm{H}_{2} \mathrm{O}(0.001 \mathrm{M})$ were 1:10, 2:10 and 3:10 for all extracts. X-ray powder diffraction (XRD), field emission scanning electron microscopy (FESEM), and ultraviolet-visible spectroscopy (UVVis) were performed to characterise the nanoparticles. The surface Plasmon resonance was located at 530, 540, and $533 \pm 2 \mathrm{~nm}$ for the synthesised Au NPs using the aqueous extract of PN, ZSC, and EUCGLO, respectively. The intensive peaks of XRD patterns indicated the crystalline nature (fcc structure) of the synthesised gold nanoparticles with an average size of $21 \sim 40 \mathrm{~nm}$. The morphology of the Au NPs was carried out by FESEM. The results showed a decline in the spherical shape of the synthesised Au NPs with a mean diameter ranged from $23.7 \sim 33.7 \pm 2 \mathrm{~nm}$.
\end{abstract}

Keywords: Piper nigrum (PN), Ziziphus spina-christi (ZSC), Eucalyptus globulus (EUCGLO), gold nanoparticles (Au NPs)

\section{Introduction}

Nanoscience and its technologies are interdisciplinary science which has achieved popularity in numerous applications in diverse disciplines owing to outstanding properties of low density, high surface area, reactivity, plasmon resonance, and many more [1]. Over the last decades, numerous reports have been dedicated to applications such as drug delivery [2], catalyst [3, 4], porous materials [5, 6], semiconductors, [7], and other different applications. Among all nanomaterials, the applications metallic nanoparticles
(NPs), have grown in demands which can be synthesised by chemical, biological, and physical methods [8]. The main drawback of the physical and chemical methods is that: they are not eco-friendly and consumable methods. In contrast, biosynthesis of NPs is an eco-friendly technique due to the involvement of many plants, microbes, and enzymes in the process [9]. The synthesis of nanoparticles by a biosynthesis method includes both intracellular and extracellular routes. The extracellular route includes several processes of reducing and capping in which those processes affect the final shape and the size of 
the nanoparticles. In addition, those processes should be clarified to control the size of the nanoparticles to be suitable for particular applications. However, standardising the methodologies and the biosynthesis' protocols is still under development, since the synthesis conditions (temperature, relative humidity, airflow rate, etc.) are different. As a consequence of nonstandardising synthesis protocols, the reliability of any procedure would be criticised in terms of repentance [10]. Production silver nanoparticles (AgNPs) and gold nanoparticles (Au NPs) with different morphologies and sizes were realised during the last decade using different routes [11-14]. Those synthetic methods involved the reduction of the ionic form of silver or gold in an appropriate medium using various reducing agents. Three characters assist the green method which is: the reducing agent, the capping agent, and the solvent. The plant extracts are particularly advantageous for the biosynthesis method of metal nanoparticles. The plant extracts gather both functions of the reducing agents and the capping agents. It also handles relatively non-toxic chemicals and non-toxic solvents (water, biological extracts) [15-17]. Green synthesis of nanoparticles includes using a wide range of plant extracts such as Capsicum annuum [16], Alfalfa [18], Amla [19], geranium [20], Aloe vera [21], and Neem [22]. In the current work, Piper nigrum (PN), Ziziphus spina-christi (ZSC), and Eucalyptus globulus (EUCGLO) extracts were adopted as a reducing and capping agents to synthesise gold nanoparticles. A comparative study on the properties of the obtained Au NPs using those plant extracts was carried out according to the molar ratio between the aqueous extract and the aqueous gold salt.

\section{Experimental}

\section{Preparation of aqueous extracts}

The preparation of the aqueous of the extract was carried out using extract to water ratio of 10:1. In a typical synthesis, $10 \mathrm{~g}$ of the dry weight of plant leaves were suspended in a $100 \mathrm{~mL}$ deionised water on vigorous stirring (500 rpm) in a $250 \mathrm{~mL}$ beaker for 2 hours at $50{ }^{\circ} \mathrm{C}$. The prepared slurry was then filtered by Whatman ${ }^{\circledR}$ qualitative filter paper, Grade 1 before dried on glass at room temperature.

\section{Synthesis of gold nanoparticles}

Gold nanoparticles were synthesised in a way reported in the literature [23] with modification. Aquatic extract (10 mL, $100 \mathrm{ppm}$ ) and aqueous gold salt $\left(\mathrm{HAuCl}_{4} \cdot 4 \mathrm{H}_{2} \mathrm{O}\right)$ were poured into a tube to obtain a gold nanoparticles mixture ( $1 \mathrm{~mL}, 100 \mathrm{ppm})$. The mixture was shaken for 5 minutes and then kept for an hour at lab temperature. After 15 minutes, the colour of the mixture started to change. The formation of reddish colour in the $\left(\mathrm{HAuCl}_{4} \cdot 4 \mathrm{H}_{2} \mathrm{O}\right)$ aqueous solution was an indicator of the reduction of the gold ions. To testify the efficiency of the extract concentration to form the nanoparticles, the experiments were repeated using 2 $\mathrm{mL}$ and $3 \mathrm{~mL}$ of the extract.

\section{X-ray diffraction}

The crystalline structure and the phase of the $\mathrm{Au}$ NPs were investigated using X-ray diffraction analysis (XRD) by employing XRD type PANalytical X'Pert PRO, Almelo, using $\mathrm{Cu}-\mathrm{K} \alpha$ radiation $(\lambda=1.54178 \AA)$ at the University of Kashan). The scan was operated to record $2 \theta$ of the pattern at range from $10^{\circ}$ to $80^{\circ}$ at the power of $40 \mathrm{kV}$ and $40 \mathrm{~mA}$. The average particle size was estimated using the Debye Scherrer method from the following equation [24]:

$\mathrm{D}=\mathrm{k} \lambda /(\mathrm{B} \cos \theta)$.

where $\theta, K, \lambda, \beta$ are the Bragg angle, the constant of the crystalline shape factor (herein is taken 0.9), wavelength, and the full width at half maximum of the sharp peaks, respectively.

\section{Scanning electron microscopy}

Scanning electron microscopy (SEM) was performed using scanning electron microscopy (type: FEG-SEM MIRA3 TESCAN, Czechia), to investigate the morphology of the nanoparticles. The technique was configured to operate at $(15.0 \mathrm{kV})$ various magnification levels. The image analysis program "ImageJ" was utilised to measure the particle size distribution. Typically, 100 nanoparticles were randomly selected from each SEM image to measure the particle size distribution. The size of the particles was measured and the particles divided into groups of $5 \mathrm{~nm}$ range.

\section{Spectroscopy measurement}

Defining the surface Plasmon resonance property (SPR) of the gold nanoparticle was achieved using a ultraviolet-visible spectrometer type Shimadzu UV-Vis 1800 at a range of wavelengths from 300 to $800 \mathrm{~nm}$.

\section{Results and Discussion}

Gold nanoparticles (Au NPs) were synthesised 
using natural materials of plants. It was first identified through the colour change into ruby-red colour during the reduction reaction (Fig. 1). The synthesised Au NPs were characterised using UV-Vis spectra, XRD and FESEM. A brief of characterisation results is given in Table 1.

Getting the benefit from the presence of the intensive surface plasmon for Au NPs in the UV-Vis absorption spectra, the technique is widely used to prove the formation of Au colloids [25]. Fig. 2(a) shows the UV spectra of Au NPs, which were synthesised using PN extract. With the increase in the concentration of the extract, a noticeable increase in the absorbance of the spectrum was accompanied by an increase in the width of the peak from 530 to $545 \mathrm{~nm}$. Fig. 2(b) shows Au
NPs UV spectra in the case of using ZSC. It has been observed that the absorption peak located at the range of $525 \sim 530 \pm 2 \mathrm{~nm}$. The shifting in the wavelength range was expected due to the reduction of Au NPs size, which is estimated in the range of $20 \sim 40 \pm 2$ $\mathrm{nm}$. The sharp peak around $535 \pm 2 \mathrm{~nm}$ gives evidence of Au NPs formation [26]. While the enhancement of the intensity might be related to the enlargement of the formed nanoparticles when gold ions number reduced in the aqueous solution. Increase the absorbance intensity of the Au NPs, which were synthesised using the extract of EUCGLO, has been noticed comparing to the other samples (Fig. 2(c)). The peak area, which is located at $525 \sim 540 \pm 2 \mathrm{~nm}$, expanded with increasing the concentration of the extract.

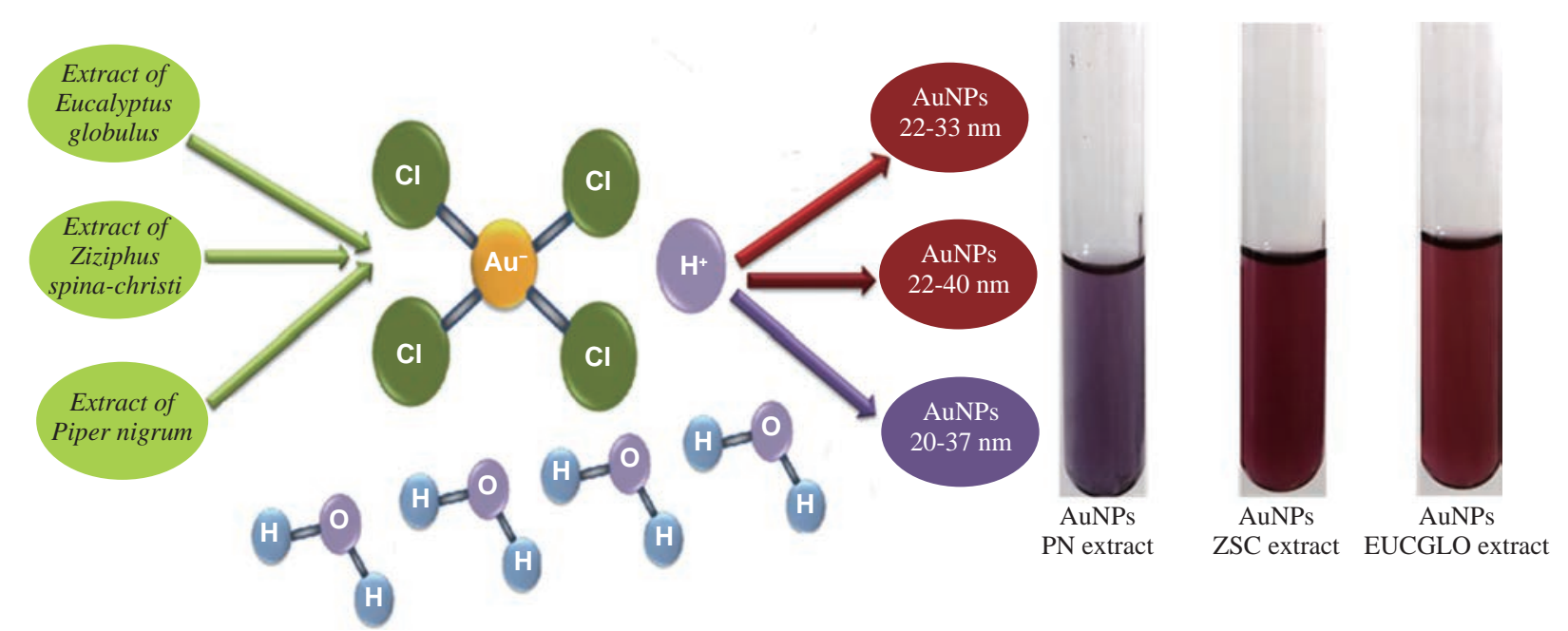

Fig. 1 Gold nanoparticles synthesised using (a) PN, (b) ZSC, and (c) EUCGLO.

Table 1 Characterisation of Au NPs synthesised using PN, ZSC, and EUCGLO

\begin{tabular}{|c|c|c|c|c|}
\hline & Volume ratio $(\mathrm{mL})$ & $\begin{array}{l}\text { XRD particle size } \\
\quad( \pm 2 \mathrm{~nm})\end{array}$ & $\begin{array}{l}\text { FESEM mean particle size } \\
\qquad( \pm 2 \mathrm{~nm})\end{array}$ & $\begin{array}{l}\text { Plasmon resonance } \\
\qquad( \pm 2 \mathrm{~nm})\end{array}$ \\
\hline \multirow{4}{*}{$\mathrm{PN}$} & 1 & 21 & 24.59 & \multirow{3}{*}{530} \\
\hline & & & & \\
\hline & 2 & 28 & 28.14 & \\
\hline & 3 & 37 & 33.7 & 545 \\
\hline \multirow{4}{*}{ ZSC } & 1 & 22 & 23.7 & \multirow{3}{*}{525} \\
\hline & & & & \\
\hline & 2 & 28 & 27.7 & \\
\hline & 3 & 33 & 32.85 & 530 \\
\hline \multirow{4}{*}{ EUCGLO } & 1 & 22 & 28.6 & \multirow{3}{*}{525} \\
\hline & & & & \\
\hline & 2 & 37 & 33.6 & \\
\hline & 3 & 40 & 32.1 & 540 \\
\hline
\end{tabular}



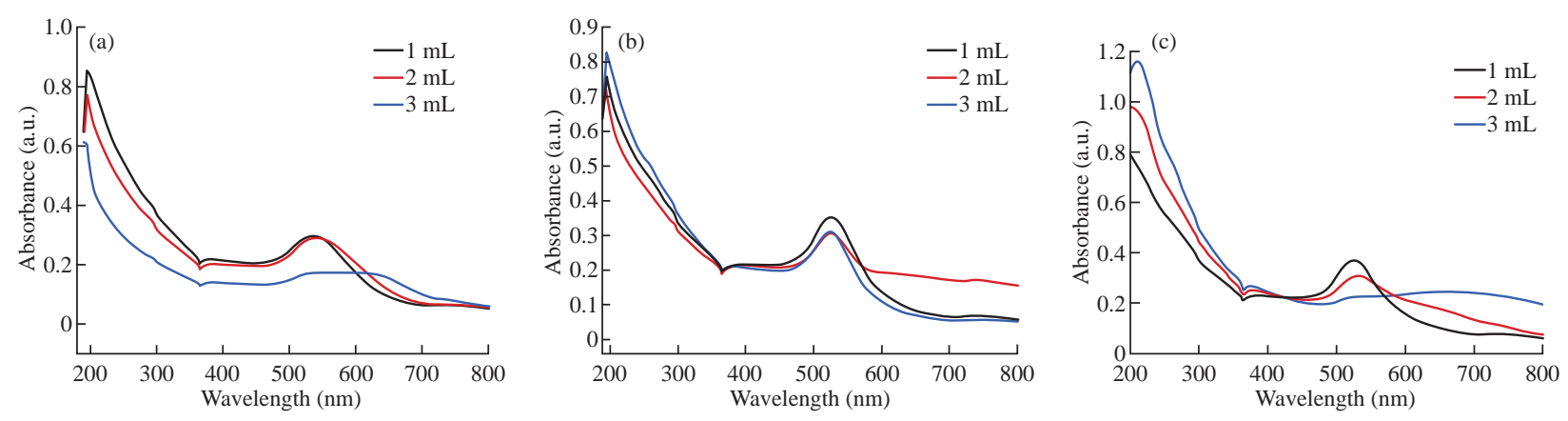

Fig. 2 The absorbance UV-visible spectra of Au NPs s synthesised using (a) PN, (b) ZSC, and (c) EUCGLO.

Fig. 3 illustrates the XRD patterns of the dried gold nanoparticles, which were deposited on the silicon substrates by the drop-casting method. The observed peaks at $38.18^{\circ}$ and $44.44^{\circ}$ are attributed to the 111 and 200 planes. It was observed that the XRD patterns of all samples do not include any other peaks related to the associated impurities. All presented peaks are characteristic of the face-cantered cubic (fcc) structure of Au. This result is in agreement with the standard JCPDS report (file no. 00-004-0784) of the XRD planes for gold. In addition, the intensity of the peak of (111) at $38.18^{\circ}$ is much stronger than those peaks of (200) at $44.44^{\circ}$ for the synthesised samples using $1 \mathrm{~mL}$ and $2 \mathrm{~mL}$ of the plant extract. While the synthesised samples using $3 \mathrm{~mL}$ have only one peak located at $38.18^{\circ}$ which could be attributed to the 200 crystallographic planes. The average crystalline size of the Au NPs (estimated by Debye-Scherrer equation) is varied for the tested samples. The particle size of the synthesised Au NPs using PN extract was in the range of $20 \sim 37 \pm 2 \mathrm{~nm}$, while the average crystallite size for the synthesised Au NPs using EUCGLO extract was in the range of $22 \sim 33 \pm 2 \mathrm{~nm}$, and ranged from $22 \sim 40$ $\pm 2 \mathrm{~nm}$ for the samples synthesised using ZSC extract.

The SEM images (Fig. 4) demonstrate the morphology of the synthesised Au NPs using different concentrations of the PN extracts. Fig. 4(a)-(d) show the image and the particle size distribution of the well- dispersed Au NPs which were synthesised using $1 \mathrm{~mL}$ of the PN extract. The particle size of the nanoparticles is distributed in a range of nanometres. However, the majority of the particles are distributed at a range from 16 to $35 \mathrm{~nm}$ with a spherical shape and the mean particle size was $24.59 \pm 2 \mathrm{~nm}$. However, some aggregated $\mathrm{Au}$ NPs were noticed. Fig. 4(b)-(e) show the SEM image and the particle size distribution of the synthesised $\mathrm{Au}$ NPs using $2 \mathrm{~mL}$ of the PN extract. The spherical shape nanoparticles have a particle size mainly distributed between 16-35 nm with a mean particle size of 28.14 $\pm 2 \mathrm{~nm}$. It is noticed that the $2 \mathrm{~mL}$ concentration of PN yields spherical shape Au NPs most of them ranged between 20-30 nm with less accumulation of the nanoparticles. While the synthesised Au NPs using $3 \mathrm{~mL}$ of the extract (Fig. 4(c)-(f)), produces larger particle in size which are distributed between 21-45 $\mathrm{nm}$, in addition, to increase the accumulation of the nanoparticles. The mean particle size was $33.7 \pm 2 \mathrm{~nm}$.

Fig. 5(a)-(d) illustrate the SEM images and the particle size distribution of the Au NPs which were synthesised using $1 \mathrm{~mL}$ of ZSC. The mean particle size is $23.7 \pm 2 \mathrm{~nm}$. However, there is a wide range of particle size distribution and more aggregation of the particles was observed. It can be seen from the SEM image and the related bar chart in Fig. 5(b)(e) that the utilising $2 \mathrm{~mL}$ of ZSC in the synthesis of $\mathrm{Au}$ NPs produced particles where the majority of it
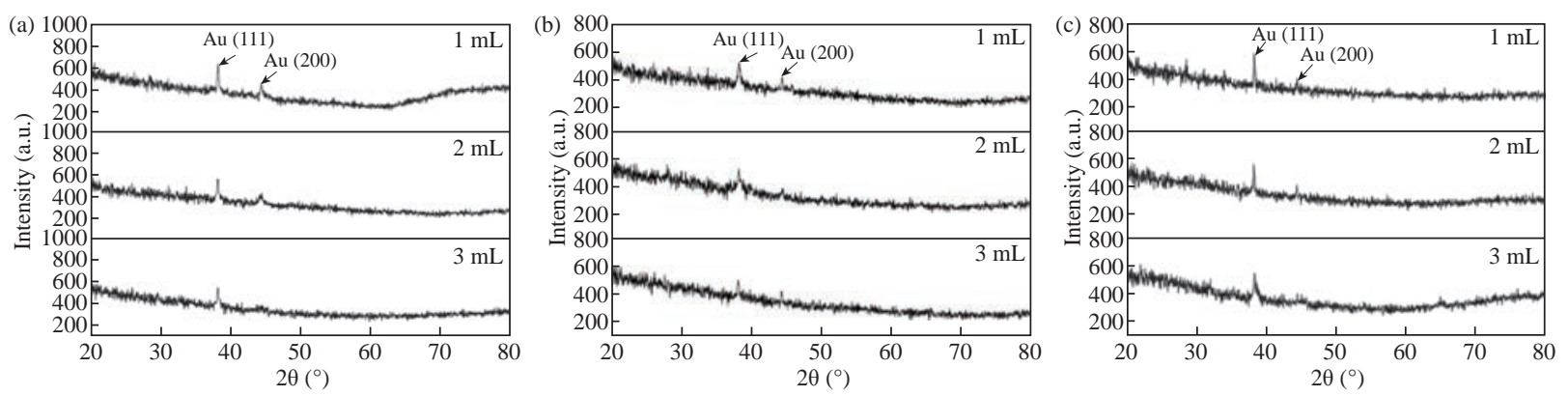

Fig. 3 The Au NPs XRD patterns of the samples synthesised using (a) PN, (b) ZSC, and (c) EUCGLO. 

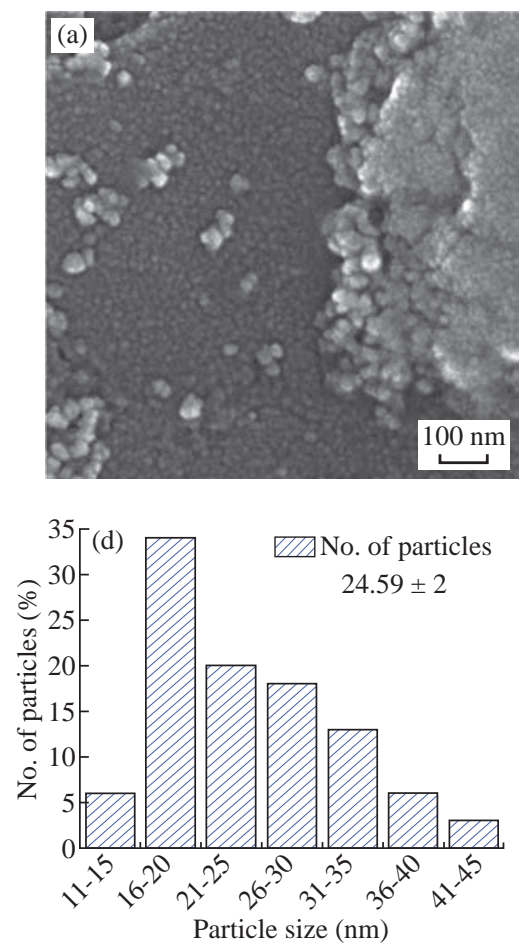
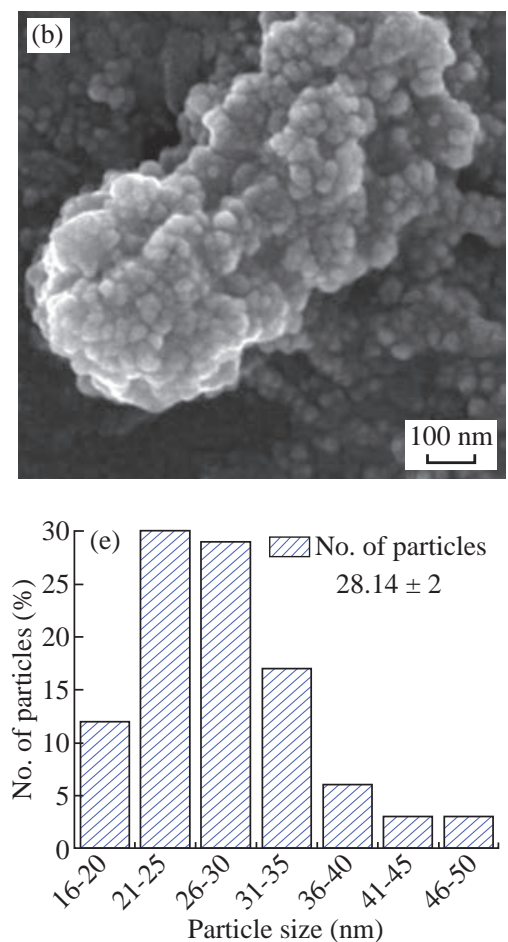
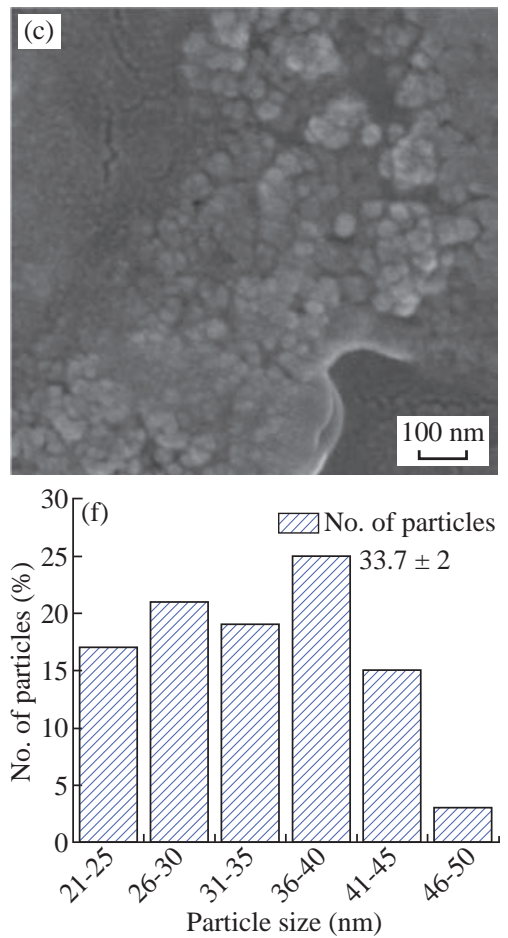

Fig. 4 SEM images of the synthesised Au NPs using PN, (a) $1 \mathrm{~mL}$, (b) $2 \mathrm{~mL}$ and (c) $3 \mathrm{~mL}$ of PN extract. (d) The particle size of nanoparticles in the image (a); (e) the particle size of nanoparticles in the image (b); (f) the particle size of nanoparticles in the image (c).
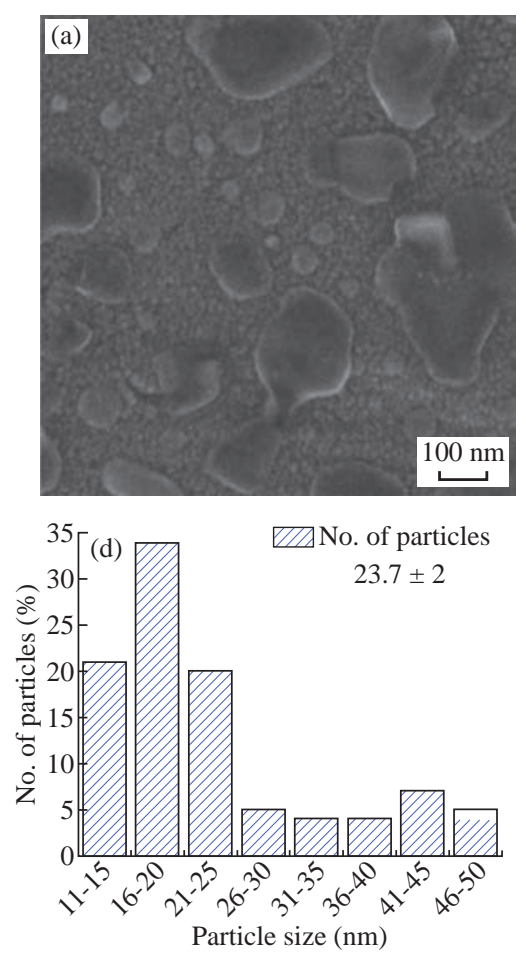
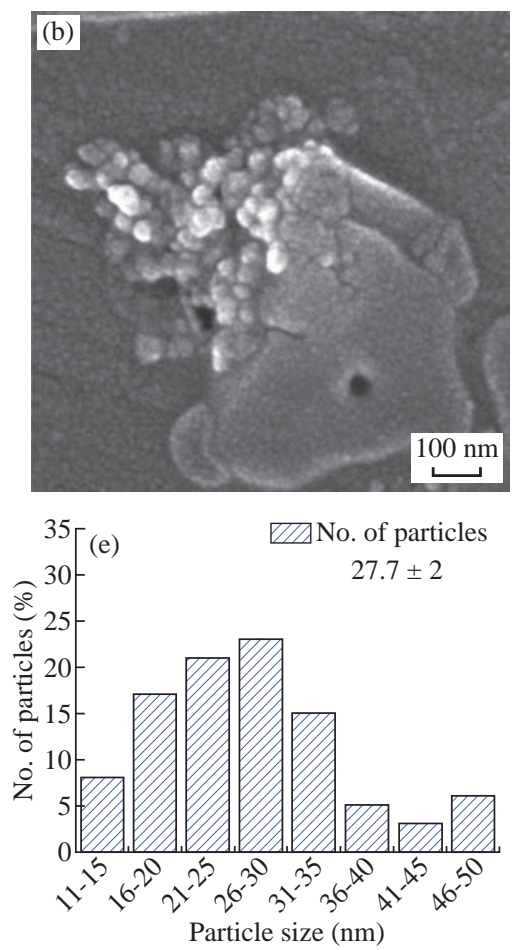
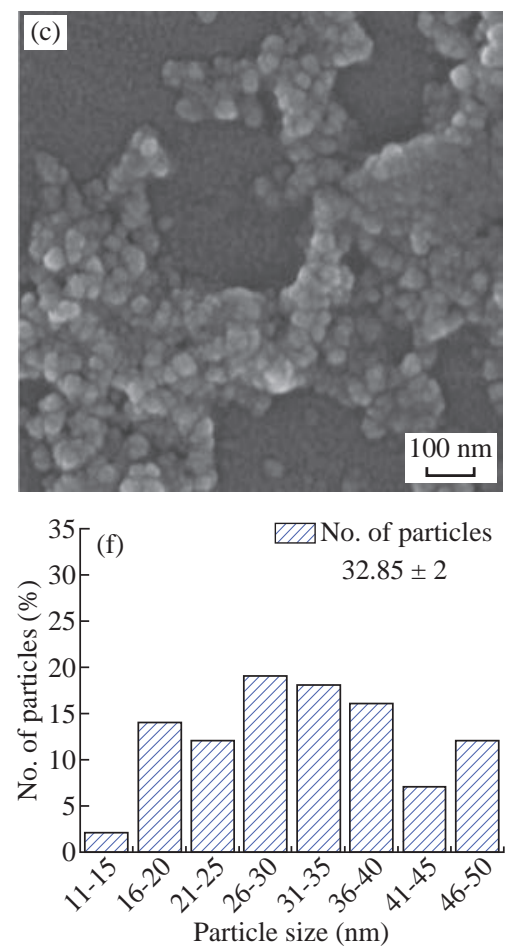

Fig. 5 SEM images of the synthesised Au NPs using ZSC, (a) $1 \mathrm{~mL}$, (b) $2 \mathrm{~mL}$ and (c) $3 \mathrm{~mL}$ of ZSC extract. (d) The particle size of nanoparticles in image (a); (e) the particle size of nanoparticles in image (b); (f) the particle size of nanoparticles in image (c).

distributed in the range between 16-35 nm. The mean diameter of the particles is $27.7 \pm 2 \mathrm{~nm}$. Furthermore, the particle size has increased again with increasing the concentration of the ZSC to $3 \mathrm{~mL}$ (Fig. 5(c)-(f)). The mean particle diameter is $32.85 \pm 2 \mathrm{~nm}$ which is quite close to that obtained result by XRD.

Fig. 6(a)-(d) illustrate the SEM images and particle size distribution of the Au NPs when $1 \mathrm{~mL}$ of EUCGLO extract has been used. The majority of the particles are distributed in the range between 21- 

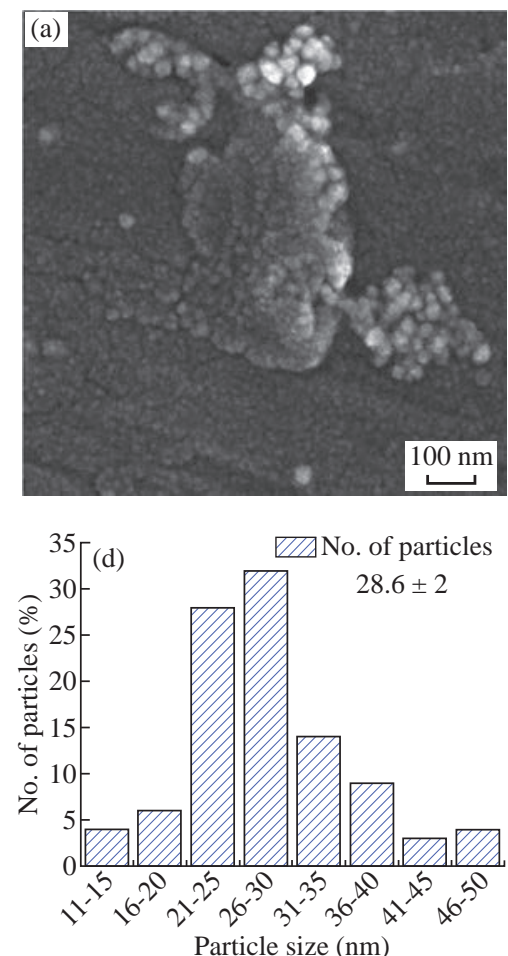
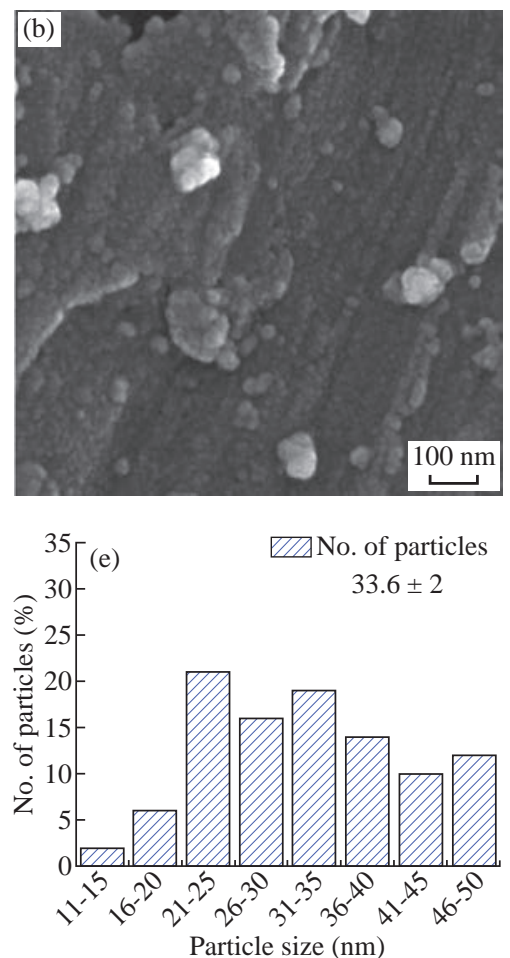
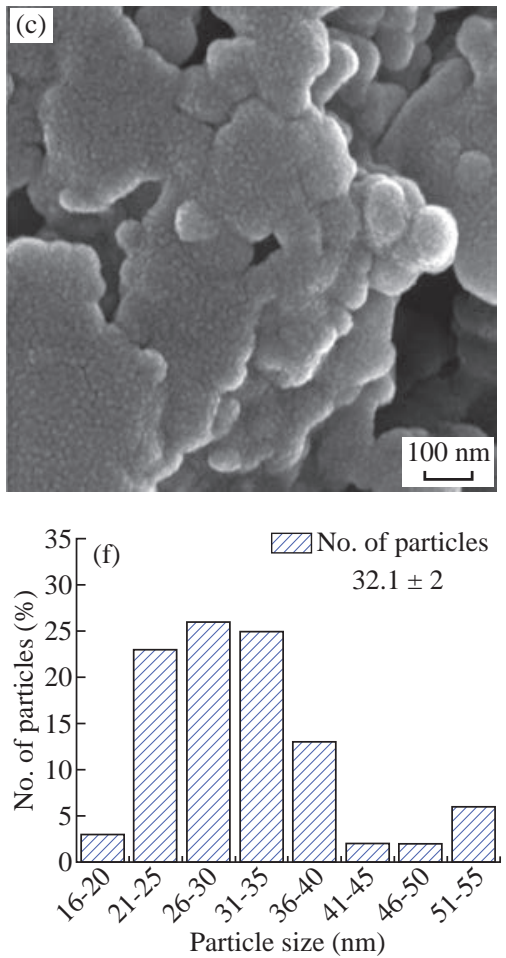

Fig. 6 SEM images of the synthesised Au NPs using Eucalyptus globulus (EUCGLO), (a) $1 \mathrm{~mL}$, (b) $2 \mathrm{~mL}$, and (c) $3 \mathrm{~mL}$ of EUCGLO extract. (d) The particle size of nanoparticles in the image (a); (e) the particle size of nanoparticles in the image (b); (f) the particle size of nanoparticles in the image (c).

$30 \mathrm{~nm}$. The mean particle size is $28.6 \pm 2 \mathrm{~nm}$. The particle size again increased as the concertation of the EUCGLO increased. That can be seen in Fig. 6(b)-(e) where the mean particle size is $33.6 \pm 2 \mathrm{~nm}$ in the case of using $2 \mathrm{~mL}$ of EUCGLO. While the mean particle size when $3 \mathrm{~mL}$ of the extract is used is $32.1 \pm 2 \mathrm{~nm}$ (Fig. 6(c)-(f)). In general, the accumulation of the nanoparticles has grown with the concentration of the extract. However, the optimum results were noticed for the PN extract, and more specifically when $2 \mathrm{~mL}$ of the extract has been used. It is noticeable that the obtained NPs particle size from FESEM microscopy is in agreement with that obtained using the $\mathrm{X}$-ray diffraction. It has been observed from the average particle size of the synthesised Au NPs suggested that the PN extract produced smaller particle size while EUCGLO extract produced the larger particle size. In addition, increase the plant extract concentrations led to produce more particles and enlarge the size especially in the case of EUCGLO. This thought to be due to the fact that EUCGLO extract is a stronger reducing agent compared to ZSC extract and PN extract, respectively.

\section{Conclusions}

Gold nanoparticles were successfully synthesised using different plant extracts (Piper nigrum, Ziziphus spina-christi, and Eucalyptus globulus). A comparative study on the properties of the Au NPs concerning the gold salt : paper extract volume ratio was carried out. Taking advantage of PN extract in the synthesis of $\mathrm{Au}$ NPs achieved a better result in terms of smaller size, uniform particle size, and crystallinity, when compared with ZSC and EUCGLO extracts. This was thought to be because Piper nigrum worked a slower reducing agent than the other extracts.

\section{Acknowledgments}

The authors would like to thank the D.P., College of science, University Of Anbar. The authors also would like to thanks Miss Leen AL-Fatyan for assistance.

\section{Conflict of Interests}

The authors declare that no competing interest exists.

\section{References}

[1] A.G. Al-Nuairi, Biosynthesis, Characterization, and evaluation of the cytotoxic effects of biologically 
synthesized silver nanoparticles from Cyperus conglomeratus root extracts on breast cancer cell line MCF-7. Biological Trace Element Research, 2020, 194(2): 560-569.

[2] D. George, P.U. Maheswari, and K. Begum, Chitosancellulose hydrogel conjugated with L-histidine and zinc oxide nanoparticles for sustained drug delivery: Kinetics and in-vitro biological studies. Carbohydrate Polymers, 2020, 236: 11.

[3] K.T. Hassan, Catalytic performance of nickel nanowires immobilized in silica aerogels for the $\mathrm{CO}_{2}$ hydration reaction. ACS Omega, 2019, 4(1): 1824-1830.

[4] J. Lu, Morphology control of nickel nanoparticles prepared in situ within silica aerogels produced by novel ambient pressure drying. Scientific Reports, 2020, 10(1): 11743.

[5] S. Ghaderi, Thermoelectric characterization of nickelnanowires and nanoparticles embedded in silica aerogels. AIP Advances, 2018, 8(6).

[6] X. Han, Bioinspired synthesis of monolithic and layered aerogels. Advanced Materials, 2018, 30(23).

[7] G.C. Fan, Enhanced photoelectrochemical immunosensing platform based on CdSeTe@CdS:Mn core-shell quantum dots-sensitized $\mathrm{TiO}_{2}$ amplified by CuS nanocrystals conjugated signal antibodies. Analytical Chemistry, 2016, 88(6): 3392-3399.

[8] G.K. Agceli, A novel approach to synthesize $\mathrm{TiO}_{2}$ nanoparticles: Biosynthesis by using Streptomyces sp. HC1. Journal of Inorganic and Organometallic Polymers and Materials, 9.

[9] H.W. Zhao, Biosynthesis of copper nanoparticles using Allium eriophyllum Boiss leaf aqueous extract; characterization and analysis of their antimicrobial and cutaneous wound-healing potentials. Applied Organometallic Chemistry, 16.

[10] O.V. Singh, Proteomics and metabolomics: The molecular make-up of toxic aromatic pollutant bioremediation. Proteomics, 2006, 6(20): 5481-5492.

[11] P. Mohanpuria, N.K. Rana, and S.K. Yadav, Biosynthesis of nanoparticles: technological concepts and future applications. Journal of Nanoparticle Research, 2008, 10(3): 507-517.

[12] K.B. Narayanan, N. Sakthivel, Biological synthesis of metal nanoparticles by microbes. Advances in Colloid and Interface Science, 2010, 156(1-2): 1-13.

[13] X.L. Zhang, Synthesis of nanoparticles by microorganisms and their application in enhancing microbiological reaction rates. Chemosphere, 2011, 82(4): 489-494.

[14] P.P. Gan, S.F.Y. Li, Potential of plant as a biological factory to synthesize gold and silver nanoparticles and their applications. Reviews in Environmental Science and Bio-Technology, 2012, 11(2): 169-206.

[15] K.N. Thakkar, S.S. Mhatre, and R.Y. Parikh, Biological synthesis of metallic nanoparticles. NanomedicineNanotechnology Biology and Medicine, 2010, 6(2): 257-
262.

[16] K. Saravanakumar, Biogenic silver nanoparticlespolyvinylpyrrolidone based glycerosomes coating to expand the shelf life of fresh-cut bell pepper (Capsicum annuum L. var. grossum (L.) Sendt). Postharvest Biology and Technology, 2020, 160: 10.

[17] T.A. Salih, In vitro scolicidal activity of synthesised silver nanoparticles from aqueous plant extract against Echinococcus granulosus. Biotechnology Reports, 2020, e00545.

[18] M. Keshavarzi, The effects of three types of alfalfa plants (Medicago sativa) on the biosynthesis of gold nanoparticles: an insight into phytomining. Gold Bulletin, 2018, 51(3): 99-110.

[19] F.S. Rosarin, Antiproliferative effect of silver nanoparticles synthesized using amla on Hep2 cell line. Asian Pacific Journal of Tropical Medicine, 2013, 6(1): 1-10.

[20] B.A. Abbasi, Plant-mediated synthesis of nickel oxide nanoparticles (NiO) via Geranium wallichianum: Characterization and different biological applications. Materials Research Express, 2019, 6(8): 15.

[21] P. Chandrababu, S. Cheriyan, and R. Raghavan, Aloe vera leaf extract-assisted facile green synthesis of amorphous $\mathrm{Fe}_{2} \mathrm{O}_{3}$ for catalytic thermal decomposition of ammonium perchlorate. Journal of Thermal Analysis and Calorimetry, 2020, 139(1): 89-99.

[22] N.D.S. Zambri, Utilization of Neem leaf extract on biosynthesis of iron oxide nanoparticles. Molecules, 2019, 24(20): 12.

[23] K.X. Lee, Green synthesis of gold nanoparticles using aqueous extract of Garcinia mangostana fruit peels. Journal of Nanomaterials, 2016, 7.

[24] A.S. Obaid, Preparation of chemically deposited thin films of CdS/PbS solar cell. Superlattices and Microstructures, 2012, 52(4): 816-823.

[25] V. Kumar, Size-dependent synthesis of gold nanoparticles and their peroxidase-like activity for the colorimetric detection of glutathione from human blood serum. Acs Sustainable Chemistry \& Engineering, 2018, 6(6): 76627675.

[26] A. Mishra, S.K. Tripathy, and S.I. Yun, Fungus mediated synthesis of gold nanoparticles and their conjugation with genomic DNA isolated from Escherichia coli and Staphylococcus aureus. Process Biochemistry, 2012, 47(5): 701-711.

Copyright $(\subset$ Ibraheem Jaleel Ibraheem, Khalil Thabit Hassan, Hameed Hussein Ali, and Ahmed Salman Obaid. This is an open-access article distributed under the terms of the Creative Commons Attribution License, which permits unrestricted use, distribution, and reproduction in any medium, provided the original author and source are credited. 